\title{
Practical Stability of Dynamic Systems with Time Delays in Terms of Two Measurements
}

\author{
Peng Chao, Yang Yuhua \\ Department of Mathematics and Physics \\ North China Electric Power University \\ Baoding, China,071003 \\ haoluzaiqianfang@163.com
}

\author{
Zhang Yan \\ Department of Mathematics and Physics \\ North China Electric Power University \\ Baoding, China,071003
}

\begin{abstract}
The paper discusses practical stability and strict practical stability of differential equation on ring neighborhood by means of Lyapunov function. The paper develops $\left(h_{0}, h\right)$ - practical stability by employing two auxiliary functions and using comparative method. Some criteria and results which are used to guarantee practical stability for differential equation are given by the method of Lyapunov function, which enables us to obtain results under weaker assumptions.
\end{abstract}

Keywords-practical stability, strict practical stability, delay differential equations, $\left(h_{0}, h\right)-$ practical stability, ring neighborhood, comparative theorem

\section{INTRODUCTION}

All manuscripts must be in English. These guidelines include complete descriptions of the fonts, spacing, and related information for producing your proceedings manuscripts. Please follow them and if you have any questions, direct them to the production editor in charge of your proceedings at Conference Publishing Services (CPS): Phone +1 (714) 821-8380 or Fax +1 (714) 761-1784.

Theory of stable in the sense of Lyapunov has been researched enough and can be widely used in concrete problems of the real world. But the stable domain or the domain of attractive is not large enough to allow the desired deviation to cancel out, thus a notion of stability is desired from practical considerations. S. Leela [1] first puts forward the definition of practical stable, and he gives a systematic study of the theory of practical stability and gives some sufficient conditions which guarantee the strict stability of the considered system. Mohapatra [2] advanced the concept of strict stable for different system .V.L.akshmilantham [3] develop the concepts of strict practical stable to delay differential equations by using Lyapunov function. Kobayashi [4] gives boundedness in differential equation with finite delay by using auxiliary function. In the paper we develop strict practical stable on ring neighborhood $D_{k}$. Significance of the paper is that $d V / d t$ is variable number outside of $D_{k}$. We research $\left(h_{0}, h\right)$ - practical stability of differential equation.

\section{MAIN RESULTS}

We consider the functional differential equation with finite delay

$$
x^{\prime}=f\left(t, x_{t}\right)
$$

$f: R_{+} \times C_{H} \rightarrow R^{n}$ is completely continuous, $C_{H}$ is a subset of the space of continuous functions $\varphi:[-h, 0] \rightarrow R^{n}, \| \varphi|=\sup | \varphi(s) \mid, \varphi \in C_{H}$, and

$0<H \leq+\infty$, Let $x(t)=x\left(t, t_{0}, \varphi\right)$ denotes a solution of (1)with initial condition $x_{t_{0}}=\varphi$. We always assume the existence of solution $x\left(t, t_{0}, \varphi\right)$ or $x_{t}$ of (1) for $t \geq t_{0}$, and $f(t, 0)=0$.

Let $U, V \quad: \quad R_{+} \times C_{H} \rightarrow R_{+} \quad, \quad \gamma: R_{+} \rightarrow R_{+} \quad, \quad$ and $g_{\lambda}: R \rightarrow R, 0<\lambda<H$ are continuous. Suppose there exists a $r_{0}>0$ such that $g_{\lambda}(r)>0$ for $0<r<r_{0}, g_{\lambda}(r) \geq \alpha_{\lambda} r$ for $r \geq r_{0}$, where $\alpha_{\lambda}>0$.

Theorem1. Suppose that

(1) $0 \leq V(t, \varphi) \leq W_{1}(\|\varphi\|)$, there are $\beta, \lambda, A$, such that

$W_{2}(|\varphi(0)|) \leq U(t, \varphi) \leq W_{3}(|\varphi(0)|), 0 \leq \beta<\lambda<A$,

$V_{(1)}^{\prime}\left(t, x_{t}\right) \leq-\gamma_{\beta}(t)$, if $\beta \leq \inf _{t-h \leq s \leq t}|x(s)|<H$

$V_{(1)}{ }^{\prime}\left(t, x_{t}\right) \leq-g_{\lambda}\left(U_{(1)}{ }^{\prime}\left(t, x_{t}\right)\right)$, if $\lambda \leq|x(t)|<H$

where $U_{(1)}{ }^{\prime}\left(t, x_{t}\right)$ is finite and locally integrable or $U\left(t, x_{t}\right)$ is absolutely continuous for any solution of (1).

(2) There exists a constant $S>0$, such that $\int_{t}^{t+s} \gamma_{\beta}(s) d s>W_{1}(A)$ uniformly in $t \in R_{+}$,

(3)Let $L=\frac{1}{2} \min \left[\alpha_{\lambda}, \inf _{\delta_{1} \leq r \leq r_{0}} g_{\lambda}(r) / r_{0}\right]$, such that

$W_{1}(\lambda)<L\left(W_{2}(A)-W_{3}(\lambda)\right)$

where $\delta_{1}=W_{2}(A)-W_{3}(\lambda) / 2(h+S)$ 
Then the zero solution of (1) is uniformly practical stability with respect $(\lambda, A)$.

Proof. For some $t_{0} \in R$ and $\varphi \in C_{H}$ with $\|\varphi\|<\lambda$, let $x(t)=x\left(t, t_{0}, \varphi\right)$ be a solution of (1) on $\left(t_{0},+\infty\right)$. We claim that $\|\varphi\|<\lambda$ implies $\|x(t)\|<A$.If it is not true, there exists a solution $x_{t}\left(t_{0}, \varphi\right)$ with $\|\varphi\|<\lambda$ and $t_{1}, t_{2}$, such that $\left|x\left(t_{1}\right)\right|=A, x\left(t_{2}\right)=\lambda,|x(t)|<A$ on $\left[t_{0}, t_{1}\right)$. Since in view of (2), we have $t_{1}-t_{2}<h+S$, for otherwise $0 \leq v\left(t_{2}+h+S\right) \leq v\left(t_{2}+h\right)-\int_{t_{2}+h}^{t_{2}+h+S} \gamma_{\lambda}(s) d s<W_{1}(A)-W_{1}(A)=0$

This is impossible.

Put $\quad Q_{1}=\left\{t \in\left[t_{2}, t_{1}\right] ; u^{\prime}(t) \geq r_{0}\right\}$

$Q_{2}=\left\{t \in\left[t_{2}, t_{1}\right] ; \delta_{1} \leq u^{\prime}(t)<r_{0}\right\}$ and $Q_{3}=\left[t_{2}, t_{1}\right] \backslash\left(Q_{1} \cup Q_{1}\right)$. Then

$$
\begin{aligned}
& W_{2}(A)-W_{3}(\lambda)=W_{2}\left(\left|x\left(t_{1}\right)\right|\right)-W_{3}\left(\left|x\left(t_{2}\right)\right|\right) \leq u\left(t_{1}\right)-u\left(t_{2}\right) \\
& \leq \int_{t_{2}}^{t_{1}} u^{\prime}(t) d t=\int_{Q_{1}} u^{\prime}(t) d t+\int_{Q_{2}} u^{\prime}(t) d t+\int_{Q_{3}} u^{\prime}(t) d t \\
& \leq \int_{Q_{1}} u^{\prime}(t) d t+r_{0} u\left(Q_{2}\right)+\delta_{1}\left(t_{1}-t_{2}\right)
\end{aligned}
$$

That is

$\int_{Q_{1}} u^{\prime}(t) d t+r_{0} u\left(Q_{2}\right) \geq \frac{1}{2}\left(W_{2}(A)-W_{3}(\lambda)\right)$

where $u\left(Q_{2}\right)$ denotes measure of $Q_{2}$. Since $v^{\prime}(t) \leq 0$, it follows that

$$
\begin{aligned}
& \quad v\left(t_{1}\right)-v\left(t_{2}\right) \leq \int_{t_{1}}^{t_{2}} v^{\prime}(t) d t \leq \int_{Q_{1}} v^{\prime}(t) d t+\int_{Q_{2}} v^{\prime}(t) d t \\
& \leq-\int_{Q_{1}} g_{\lambda}\left(u^{\prime}(t)\right) d t-\int_{Q_{2}} g_{\lambda}\left(u^{\prime}(t)\right) d t \\
& \leq-\alpha_{\lambda} \int_{Q_{1}} u^{\prime}(t) d t-\inf _{\delta_{1 \leq} \leq r_{0}} g_{\lambda}(r) u\left(Q_{2}\right) \\
& \text { Since } \quad L=\frac{1}{2} \min \left[\alpha_{\lambda}, \inf _{\delta_{1} \leq r_{0}} g_{\lambda}(r) / r_{0}\right]
\end{aligned}
$$$$
\text { thus } v\left(t_{1}\right)-v\left(t_{2}\right) \leq-L\left(W_{2}(A)-W_{3}(\lambda)\right) \text {. }
$$

In view of (3), then we have

$0 \leq v\left(t_{1}\right) \leq v\left(t_{2}\right)-L\left(W_{2}(A)-W_{3}(\lambda)\right)<v\left(t_{2}\right)-W_{1}(\lambda)<0$

That is a contradiction. Hence $|x(t)|<A_{\text {on }}\left[t_{0},+\infty\right)$, This proves conclusion.

Let

$V(t, \varphi), U(t, \varphi) \in C\left[R_{+} \times C_{H}, R_{+}\right], \quad V(t, \varphi), U(t, \varphi)$ is locally lipschitz in $\varphi, \lambda_{i}(r): R_{+} \rightarrow R_{+}, i=1,2$, and $g_{\gamma}(r): R \rightarrow R$ are continuous. Suppose there exist constants $\alpha 、 r_{0}>0$ such that $g_{\gamma}(r)>0$ for $r \leq r_{0}$, $g_{\gamma}(r) \geq \alpha r$ for $r \geq r_{0}$.

$$
\text { Let } w_{i}(s) \in K \quad(i=1,2,3) .
$$

Theorem 2 Assume that

$$
\begin{aligned}
& 0 \leq V(t, \varphi) \leq w_{1}(\|\varphi\|), \\
& w_{2}(|\varphi(0)|) \leq U(t, \varphi) \leq w_{3}(|\varphi(0)|) ;
\end{aligned}
$$

(2) $0<\lambda<A$ is given, the functions

$$
\begin{aligned}
& V(t, x(t)), \lambda_{i}(t) \quad(i=1,2) \text { are such that } \\
& \left.D^{+} V(t, u(t), \xi)\right|_{(1)} \leq-\lambda_{1}(t) \text { if } \lambda \leq \inf _{t-h \leq s<t}|x(t)|<A \\
& \left.D^{+} V(t, u(t), \xi)\right|_{(1)} \leq-g_{\lambda}\left(U_{(1)}^{\prime}\left(t, x_{t}, \xi\right)\right)+\lambda_{2}(t) \\
& \text { If } \lambda \leq|x(t)|<A
\end{aligned}
$$

Where $U_{(1)}^{\prime}\left(t, x_{t}\right)$ is finite and locally integrable or $U\left(t, x_{t}\right)$ is absolutely continuous for any solution of equation (1).

(3) there exists a constant $c$ such that

$\int_{t}^{t+h} \lambda_{2}(\xi) d \xi \leq c^{\text {uniformly }} t \in R_{+}$.

(4) there exists a constant $S>0$ such that

$\int_{t}^{t+S h} \lambda_{1}(\xi) d \xi>c+1+w_{1}(A)$ and

$\alpha\left[w_{2}(A)-w_{3}(\lambda)-r_{0}(S+1) h\right]>(S+2) c+w_{1}(A)$ Then the solutions $x\left(t, t_{0}, \varphi\right)$ of system (1) is uniformly practical stability with respect to $(\lambda, A, \sigma)$.

Proof. $\quad V(t)=V\left(t, x\left(t+\xi, \tau_{0}, \phi\right)-x\left(t, t_{0}, \varphi\right)\right) \quad$ and $U(t)=U(t, y(t)-x(t)) \quad$ for $\quad$ any $\varphi \in C_{H}$, $x(t)=x\left(t, t_{0}, \varphi\right)$, and $\|\phi-\varphi\|<\lambda,\left|\tau_{0}-t_{0}\right|<\sigma$. Choose a constant $M: 0<M<A$ such that

$$
\alpha\left[w_{2}(M)-w_{3}(\lambda)-r_{0}(S+1) h\right]>(S+2) c+w_{1}(A)
$$

First, we shall show $V(t)<w_{1}(M)$ on $\left[t_{0},+\infty\right)$ for any $\|\phi-\varphi\|<\lambda,\left|\tau_{0}-t_{0}\right|<\sigma$.If this is not true, because of condition (1) of theorem 2, we have $V\left(t_{0}\right) \leq w_{1}(\|\phi-\varphi\|)<w_{1}(\lambda)<w_{1}(M)$. So there is ${ }^{\mathrm{a}} t_{1} \geq t_{0}$ such that $w_{1}(M)=V\left(t_{1}\right) \leq w_{1}\left(\left\|x_{t_{1}}-y_{t_{1}}\right\|\right)$ and 
$V(t)<w_{1}(M)^{\text {on }}\left[t_{0}, t_{1}\right)^{. \text {It follows }}\left\|x_{t_{1}}-y_{t_{1}}\right\| \geq M$. Which implies $\left|x\left(t_{2}\right)-y\left(t_{2}\right)\right| \geq M$ for $t_{2} \in\left[t_{1}-h, t_{1}\right]$.From continuous of solutions of equation (1), there is a constant $t_{3} \in\left[t_{0}, t_{2}\right) \quad$ such that $\quad\left|x\left(t_{3}\right)-y\left(t_{3}\right)\right|=\lambda \quad$ and $\lambda<|x(t)-y(t)|<M \quad$ on $\left[t_{3}, t_{2}\right]$.Either $\quad(\quad \mathrm{i} \quad)$ $t_{2}>t_{3}+(S+1) h^{\text {or (ii) }} t_{2} \leq t_{3}+(S+1) h$.

If ( i ) holds, then

$$
\begin{gathered}
w_{1}(M)=V\left(t_{1}\right) \leq V\left(t_{2}\right)+\int_{t_{2}}^{t_{1}} \lambda_{2}(t) d t \leq V\left(t_{2}\right)+c \\
\leq V\left(t_{3}+h\right)+\int_{t_{3}+h}^{t_{2}} V^{\prime}(t) d t+c \\
\leq V\left(t_{3}+h\right)-\int_{t_{3}+h}^{t_{2}} \lambda_{1}(\xi) d \xi+c \\
\leq w_{1}(M)-\int_{t_{3}+h}^{t_{2}} \lambda_{1}(\xi) d \xi+c \leq w_{1}(M)-c-1+c<w_{1}(M)
\end{gathered}
$$

It leads to a contradiction.

If ( ii ) holds, then

$$
\begin{gathered}
w_{1}(M)=V\left(t_{1}\right)=V\left(t_{2}\right)+\int_{t_{2}}^{t_{1}} V^{\prime}(t) d t \leq V\left(t_{2}\right)+c \\
\leq V\left(t_{3}\right)+\int_{t_{3}}^{t_{2}} V^{\prime}(t) d t+c \\
\leq V\left(t_{3}\right)-\int_{t_{3}}^{t_{2}} g\left(u^{\prime}(t)\right) d t+\int_{t_{3}}^{t_{2}} \lambda \lambda_{2}(\xi) d \xi+c \\
\leq w_{1}(M)-\int_{t_{3}}^{t_{2}} g\left(u^{\prime}(t)\right) d t+(S+2) c
\end{gathered}
$$

Put $Q=\left\{t \mid U^{\prime}(t)>r_{0}, t \in\left[t_{3}, t_{2}\right\}\right.$.Then

$$
\begin{aligned}
& w_{2}(M)-w_{3}(\lambda) \leq u\left(t_{2}\right)-u\left(t_{3}\right)=\int_{t_{2}}^{t_{3}} U^{\prime}(t) d t \\
\leq & \int_{Q} U^{\prime}(t) d t+r_{0}\left(t_{2}-t_{3}\right) \leq \int_{Q} U^{\prime}(t) d t+r_{0}(S+1) h
\end{aligned}
$$

or

$$
\int_{Q} U^{\prime}(t) d t \geq w_{2}(M)-w_{3}(\lambda)-r_{0}(S+1) h
$$

From condition (4) of theorem 2 we obtain

$$
\begin{aligned}
& w_{1}(M)=V\left(t_{1}\right) \leq w_{1}(M)-\alpha \int_{t_{3}}^{t_{2}} U^{\prime}(t) d t+(S+2) c \\
& \leq w_{1}(M)-\alpha\left[w_{2}(M)-w_{3}(\lambda)-r_{0}(S+1) h\right]+(S+2) c \\
& <w_{1}(M)-(S+2) c+(S+2) c=w_{1}(M)
\end{aligned}
$$

It leads to a contradiction.

It follows $V(t)<w_{1}(M)$ on $\left[t_{0},+\infty\right)$.
We claim that $\|\phi-\varphi\|<\lambda,|\xi|<\sigma$

Implies $|x(t)-y(t)|<A^{\prime} t \geq t_{0}$.If the claim is not true, there exists $t_{4} \geq t_{0}$ such that $\left|x\left(t_{4}\right)-y\left(t_{4}\right)\right|=A$ and $|x(t)-y(t)|<A^{\text {on }}\left[t_{0}, t_{4}\right)$. Then due to $\|\phi-\varphi\|<\lambda$, we can find $t_{5} \in\left[t_{0}, t_{4}\right)$, such that $\left|x\left(t_{5}\right)-y\left(t_{5}\right)\right|=\lambda$ and $\lambda<|x(t)-y(t)|<A^{\text {on }}\left(t_{5}, t_{4}\right)$. Then

$$
\begin{aligned}
& V\left(t_{4}\right) \leq V\left(t_{5}\right)+\int_{t_{5}}^{t_{4}-h} V^{\prime}(t) d t+\int_{t_{4}-h}^{t_{4}} V^{\prime}(t) d t \\
& \leq V\left(t_{5}\right)+\int_{t_{5}}^{t_{4}-h} V^{\prime}(t) d t+c \\
& \text { If }_{t_{4}}>t_{5}+(S+1) h, \text { we have } \\
& V\left(t_{4}\right) \leq w_{1}(M)-\int_{t_{5}}^{t_{5}+S h} \lambda_{1}(\xi) d t+c \\
& \quad<w_{1}(M)-\left[c+1+w_{1}(A)\right]+c<0
\end{aligned}
$$

This contradicts to condition (1) of theorem 2 .

If $t_{4} \leq t_{5}+(S+1) h$, we have

$$
\begin{aligned}
& V\left(t_{4}\right)=V\left(t_{5}\right)+\int_{t_{5}}^{t_{4}} V^{\prime}(t) d t \\
& \leq V\left(t_{5}\right)-\int_{t_{5}}^{t_{4}} g\left(u^{\prime}(t)\right) d t+\int_{t_{5}}^{t_{5}+(S+1) h} \lambda_{2}(\xi) d \xi \\
& \leq w_{1}(M)-\alpha\left[w_{2}(A)-w_{3}(\lambda)-r_{0}(S+1) h\right]+(S+1) c \\
& \leq w_{1}(M)-(S+2) c+(S+1) c-w_{1}(A)<0
\end{aligned}
$$

This contradicts to condition (1) of theorem 2. The contradiction obtained proves that solutions of system (1) are uniformly practical stability with respect to $(\lambda, A)$. The proof is complete.

We consider the differential system

$$
x^{\prime}=f(t, x), x\left(t_{0}\right)=x_{0}, t_{0} \in R_{+}
$$

where $f \in C\left[R_{+} \times R^{n}, R^{n}\right]$.

The scalar differential equation

$$
u^{\prime}=g(t, u), u\left(t_{0}\right)=u_{0} \geq 0
$$

Where $g \in C\left[R_{+}{ }^{2}, R_{+}\right]$and $g(t, u)$ is quasimonotone nondecreasing in $u$.

Theorem3. Assume that

(i) $0<\lambda<A$;

(ii) $h_{0}, h \in \Gamma$ and $h_{0}$ is finer than $h$ i.e.h $(t, x) \leq \Phi\left(h_{0}(t, x)\right), \Phi \in K$

Whenever $\quad h_{0}(t, x) \leq \lambda$

$\Gamma=\left\{h \in C\left[R_{+} \times R^{n}, R_{+}\right]: \inf _{(t, x)} h(t, x)=0\right\}$ 
(iii) $V \in C\left[R_{+} \times R^{n}, R_{+}\right], V(t, x)$ is locally Lipschitzian in $\mathrm{x}$ and satisfies

(1) $b(h(t, x)) \leq V(t, x) \leq a(h(t, x))$ if $\lambda<h(t, x)<A$

(2) $D^{+} V(t, x) \leq g(t, V(t, x)),(t, x) \in S(h, A)$, where $g \in C\left[R_{+}^{2}, R\right]$ and $S(h, A)=\left\{(t, x) \in R_{+} \times R^{n}: \lambda_{1}<h(t, x)<A\right\}, \lambda_{1}<\lambda$.

(iv) $\Phi(\lambda)<A$ and $a(\lambda)<b(A)$ hold.

Then the practically stable properties of (3) imply the corresponding $\left(h_{0}, h\right)$ - practically stable properties of the system (2).

Proof. Suppose that the equation (3) is practically stable with respect to $(a(\lambda), b(A))$,so that we have $u_{0}<a(\lambda)$ implies $u\left(t, t_{0}, u_{0}\right)<b(A) t \geq t_{0}$. Then we claim that the system (2) is $\left(h_{0}, h\right)$ - practically stable with respect to $(\lambda, A)$. If this is not true, then there would exist $t_{2}>t_{1}>t_{0}$ and a solution $x(t)=x\left(t, t_{0}, x_{0}\right)$ such that

$$
h_{0}\left(t_{0}, x_{0}\right)<\lambda, h\left(t_{1}, x\left(t_{1}\right)\right)=\lambda_{1}, h\left(t_{2}, x\left(t_{2}\right)\right)=A \quad,
$$
$t_{1}<t<t_{2}$.

Since in view of conditions (ii) and (iv), we have $h\left(t_{0}, x_{0}\right) \leq \Phi\left(h_{0}\left(t_{0}, x_{0}\right)\right)<\Phi(\lambda)<A$

Hence, In view of (iv) and (2), we get

$V(t, x(t)) \leq r\left(t, t_{1}, V\left(t_{1}, x\left(t_{1}\right)\right)\right), t_{1} \leq t \leq t_{2}$.

where $r\left(t, t_{1}, u_{0}\right)$ is the maximal solution of $(3)$, $r\left(t_{1}, t_{1}, u_{0}\right)=u_{0}=V\left(t_{1}, x\left(t_{1}\right)\right)$.

$b(A)=\lim _{t \rightarrow t_{2}} b(h(t, x(t)))=b\left(h\left(t_{2}, x\left(t_{2}\right)\right)\right) \leq V\left(t_{2}, x\left(t_{2}\right)\right)$

$\leq r\left(t_{2}, t_{1}, V\left(t_{1}, x\left(t_{1}\right)\right)\right) \leq r\left(t_{2}, t_{1}, a\left(\lambda_{1}\right)\right)<b(A)$.

That is a contradiction. This proves $\left(h_{0}, h\right)$ - practical stability properties of the system (2).

Theorem4. Suppose that

(1) There exists sequence of positive number $\left\{r_{k}\right\}$ and constants $\lambda, A$,

$0<\lambda<A$

$$
\begin{gathered}
l=\inf _{\|x\|=A} V(t, x)>0, \lim _{k \rightarrow \infty} r_{k}=l, p=\sup _{\|x\|=\lambda} V(t, x) . \\
b(|x(t)|) \leq V(t, x(t)), \quad b(r) \in K .
\end{gathered}
$$

$D_{k}=\left\{x \mid 0 \leq r_{k}-\eta_{k} \leq V(t, x(t)) \leq r_{k}\right\} \quad(k=1,2, \ldots) \quad$ such that

$$
D^{+} V \leq 0,|x(t)| \geq \lambda, x \in D_{k}
$$

(3) For any $0<\lambda_{1}<\lambda$, there exists sequence of positive number $\left\{\xi_{k}\right\}$ and constant $A_{2}$, such that $A_{2}<\lambda_{1}$,

$$
\begin{gathered}
\lim _{k \rightarrow \infty} \xi_{k}=m, \quad m=\sup _{\|x\|=A_{2}} V(t, x)>0 . \\
b_{1}(|x(t)|) \leq V_{1}(t, x(t))
\end{gathered}
$$

Let

$D_{k}^{\prime}=\left\{x \mid \xi_{k} \leq V_{1}(t, x(t)) \leq \xi_{k}+l_{k}\right\}(k=1,2, \ldots)$ such that

$$
D_{-} V_{1} \geq 0,|x(t)|<\lambda_{1}, \quad x \in D_{k}^{\prime}
$$

Then the system (1) is strict practical stable.

Proof. Since in view of (1) $\lim _{k \rightarrow \infty}=l$, there exists $t_{1}, t_{2},\left|x\left(t_{1}\right)\right|=\lambda,\left|x\left(t_{2}\right)\right|=A$. Since $P \leq r_{k} \leq l$, thus there exists $K$ and $t_{3}, t_{4} \in\left[t_{1}, t_{2}\right]$, such that

$$
\begin{aligned}
& V\left(t_{3}, x\left(t_{3}\right)\right)=r_{K}-\eta_{K}, V\left(t_{4}, x\left(t_{4}\right)\right)=r_{K} \\
& \text { So } \\
& V\left(t_{3}, x\left(t_{3}\right)\right)<V\left(t_{4}, x\left(t_{4}\right)\right)
\end{aligned}
$$

On the other hand, $r_{K}-\eta_{K} \leq V(t, x) \leq r_{K}$, because of condition (2), So $V(t, x)$ is nonincrease, $V\left(t_{4}, x\left(t_{4}\right)\right) \leq V\left(t_{3}, x\left(t_{3}\right)\right)$. This is contradicting to (5). Thus the solution is practical stability.

In view of (3), $\lim _{k \rightarrow \infty} \xi_{k}=m$ there exists $t_{5}, t_{6}$, $\left|x\left(t_{5}\right)\right|=\lambda_{1},\left|x\left(t_{6}\right)\right|=A_{2}$.

There exists $K^{\prime}, t_{7}, t_{8} \in\left[t_{5}, t_{6}\right] \quad$ such that, $t_{7}<t_{8}, V_{1}\left(t_{7}, x\left(t_{7}\right)\right)=\xi_{K^{\prime}}+l_{K^{\prime}}$,

$$
\begin{aligned}
& V_{1}\left(t_{8}, x\left(t_{8}\right)\right)=\xi_{K^{\prime}}, \text { So } \\
& V_{1}\left(t_{8}, x\left(t_{8}\right)\right)<V_{1}\left(t_{7}, x\left(t_{7}\right)\right)
\end{aligned}
$$

On the other hand, since in view of (3), we have $V_{1}\left(t_{7}, x\left(t_{7}\right)\right) \leq V_{1}\left(t_{8}, x\left(t_{8}\right)\right)$, this is contradicted to (6), so the solution is strict practical stability.

\section{REFERENCES}

[1] S Leela and A. A. Martynyuk Practical Stable of Nonlinear systems [M], World Scientific Publishing Co.Pet.Ltd, 1990.

[2] V.Lakshmikantham; R. N. Mohapatra, Strict stable of differential equations, Nonlinear Analysis [M], 1989.

[3] V. Lakshmikantham, Strict practical stable of delay differential equation, Applied Mathematics and computation [J], 2001.

[4] KatsumasaKobayashi, Stable and Boundedness in functional differential equations with finite delay [J], 2002.

[5] LIAO Xiao-xin. Mathematical theory and applications of stability [M], HuaZhong Normal University Press, 2001.

[6] ZHENG Da-zhong.. Linear System Theory [M], Beijing: Tsinghua University Press, 1992 